\author{
Cedo M Bagi ${ }^{1 \star}$, Edwin Berryman ${ }^{1}$, \\ Chang-Ning Liu ${ }^{2}$, Isabela B Bagi ${ }^{3}$, \\ David E Zakur ${ }^{1}$ and Catharine J \\ Andresen $^{1}$ \\ ${ }^{1}$ Comparative Medicine, Global Science \& \\ Technology, PGRD, Pfizer Inc., Groton, CT, UK \\ ${ }^{2}$ Investigative Toxicology, DSRD, Pfizer Inc., Groton, \\ CT, UK \\ ${ }^{3}$ The College of Holy Cross, Worchester, MA, UK
}

Dates: Received: 04 April, 2016; Accepted: 20 April, 2016; Published: 21 April, 2016

*Corresponding author: Cedo M Bagi, M.D., Ph.D, Pfizer Global Research \& Development, Global Science \& Technology, 100 Eastern Point Road, Groton, CT 06340, Tel: (860) 715-6465; E-mail: cedo.bagi@pfizer.com

www.peertechz.com

Keywords: OsteoarthritisL; Zoledronic acid; PTH; Dynamic weight bearing; Cortical bone strength; $\mu \mathrm{CT}$
Research Article

\section{Antiresorptive and Anabolic Bone Therapy Does not Improve Weight Bearing Capacity and Bone Strength in OA Rats}

\section{Introduction}

Osteoarthritis (OA) of the knee is the most common type of arthritis, and as such, it is the main cause of pain and disability in the elderly $[1,2]$. The risk factors related to OA are numerous and include past joint trauma or repeated micro-trauma, advanced age, obesity, nutritional factors, female gender, occupation, joint deformities, muscle weakness and genetics [3]. Out of all patients diagnosed with OA, approximately $12 \%$ have clear evidence of trauma prior to the onset of symptomatic disease [4]. The avascular nature of articular cartilage limits its regenerative and healing capacity; therefore, patients with post-traumatic OA (PTOA) heavily depend on external treatments designed to minimize damage to the joint structures and support the healing process. There is also a clear need to reestablish joint functionality in these patients because mechanical loads are critical to maintaining the health of the articular cartilage, bone, and muscles $[5,6]$. The pain caused by trauma, damaged tissue, and subsequent inflammation often creates a vicious cycle that starts with decreased physical activity and leads to atrophy of the joint and ultimately, physical debilitation $[7,8]$. Ample preclinical and clinical evidence suggests that physiologic loads are not only beneficial but necessary for the health of the joint [9-11]. The incapacity of the osteoarthritic limb to withstand physiologic loads is a critical element in OA pathophysiology; thus, the reestablishment of mechanical function through moderate exercise is recommended for the management of OA patients [12,13]. Because no effective therapies that specifically target chondrocytes exist, clinical guidelines recommend both pharmacological and nonpharmacological approaches to relieve the symptoms of OA [14]. Both bone and cartilage turnover are deemed critical for the health of synovial joints, hence the use of both antiresorptive and anabolic bone agents could be used to treat OA symptoms $[15,16]$. Preclinical data overwhelmingly indicate that antiresorptive $[17,18]$ and anabolic $[19,20]$ therapies curb progression of OA in animal models, however, clinical results fail to demonstrate clear benefits of these therapies to OA patients despite the fact that these drugs have been in clinical use for many years $[21,22]$.

A unilateral medial meniscectomy (MM) in rats results in the progressive degeneration of the articular cartilage with subsequent sclerosis and osteophyte formation, which further limit joint function [23]. The recent study conducted by our group showed no significant beneficial effects of either antiresorptive or anabolic therapy on the key denominators of $\mathrm{OA}$, including cartilage damage, osteophyte formation, osteosclerosis and joint functionality [24]. Although our study results aligned with the clinical data, we did not expect a "no-effect" outcome, particularly because the vast majority of published preclinical data shows that bone-targeting drugs have at least moderate efficacy in animal models of OA [17-20]. To further elucidate the possible reasons for the abovementioned discrepancy 
and to further extrapolate the ability of bone-targeted therapy to improve joint functionality and bone strength, we evaluated the cortical bone geometry and cortical bone strength of the femurs of injured (underloaded) and contralateral legs in a rat model of PTOA.

\section{Methods}

\section{Animals and management}

Four-month-old male Lewis rats (Charles River Laboratories, Portage, MI, USA) weighing $350 \mathrm{~g}$ were used in this study. All in vivo procedures were approved by the Institutional Animal Care and Use Committee (IACUC) at Pfizer and were performed in accordance with the Guidance [25]. The rats were pair-housed in ventilated cages (Innovive Inc., San Diego, CA, USA) in a temperature- and humidity-controlled room on a regular 12-hour light/dark cycle. Irradiated LabDiet ${ }^{\mathrm{TM}} 5053$ (Purina, Richmond, IN, USA) and water were provided ad libitum. The rats were acclimated for one week prior to their use in the study. A total of 48 rats were used for the 10 -week study, with 12 rats per group. One group of 12 rats received sham surgery; the remaining 36 rats underwent MM surgery. The four study groups were as follows: a sham control (Sham), an MM vehicle-treated $(\mathrm{MM} / \mathrm{Veh})$, an $\mathrm{MM}+$ zoledronate $(\mathrm{MM} / \mathrm{Zol})$ and an $\mathrm{MM}+\mathrm{PTH}(\mathrm{MM} / \mathrm{PTH})$

\section{Surgery}

The rats were anesthetized using isoflurane. One dose of carprofen (Pfizer Animal Health, New York, NY, USA) and sustained-release buprenorphine (Zoopharm, Windsor, CO, USA) were administered prior to surgery for analgesic coverage. In the sham group, a surgical approach to the medial collateral ligament on the right hind limb was completed by cutting the skin and leaving the medial collateral ligament intact. In the surgery groups, MM was performed by fully transecting both the medial collateral ligament and the medial meniscus of the right hind limb, followed by closure in 2 layers using absorbable sutures [23].

\section{Dosing and bone labeling}

The rats in the sham and MM/Veh control groups received vehicle (sterile water) at $1 \mathrm{~mL} / \mathrm{kg}$ subcutaneously (sc) 5 days/week starting on the day of surgery. The rats in the MM/Zol group received zoledronic acid (Zol; Sargent Pharmaceuticals 25021-801, Schaumburg, IL, USA) at $100 \mu \mathrm{g} / \mathrm{kg}$ sc twice/week [16], and the rats in the MM/PTH group received human PTH (hPTH 1-34; Sigma-Aldrich, P3796, St. Louis, MO, USA) at $40 \mu \mathrm{g} / \mathrm{kg}$ sc five times/week [20] starting on the day of surgery. To label the actively mineralized bone surfaces calcein (Sigma-Aldrich Cat\# C-0875) at $10 \mathrm{mg} / \mathrm{kg}(3.3 \mathrm{~mL} / \mathrm{kg})$ was injected 13 days before necropsy and alizarin (Sigma-Aldrich Cat\# A-5533) at $30 \mathrm{mg} / \mathrm{kg}(3.0 \mathrm{~mL} / \mathrm{kg}) 3$ days before necropsy.

\section{Body weight, sample collection}

Body weight was recorded twice weekly throughout the study. At the end of the 10-week study, both hind legs were carefully harvested and wrapped in saline-soaked gauze and frozen at $-20^{\circ} \mathrm{C}$ for ex vivo imaging, mechanical testing and histological analyses.

\section{Dynamic weight bearing}

Dynamic weight-bearing (DWB) measurements were obtained before surgery, at week 5 and before euthanasia to assess the effects of surgery on the weight-bearing capacity of the hind and front legs using a BIO-SWB-R model (Bioseb, Boulogne, France, version 1.3) and method described earlier [26].

\section{Radiology}

All of the knee samples were X-rayed with a Faxitron Model MX20 specimen scanner (Faxitron Bioptics LLC., Tucson, AZ, USA) using exposure time of $12-18 \mathrm{sec}$ at $31-35 \mathrm{kV}$ and $3 \mathrm{x}$ magnifications to inspect the bone samples possible abnormalities.

\section{$\mu C T$ and EPIC $\mu C T$ measurements}

Pre-contrast scans of all the tibias were obtained using the MicroCT $100^{\circ}$ computed tomography system (Scanco Medical, Bassersdorf, Switzerland) with the following parameters: 800 slices, $10-\mu \mathrm{m}$ resolution, a total scanned area of $8.0 \mathrm{~mm}^{2}$, and source energy of $70 \mathrm{kVp}, 115 \mu \mathrm{A}$ at $8 \mathrm{~W}$ to capture the entire proximal tibia section. The tibias were then incubated in the Hexabrix solution and scanned using a previously described method [24,27]. Post-soak scanning of the right tibia was performed using source energy of $55 \mathrm{kVp}, 145 \mu \mathrm{A}$ at $8 \mathrm{~W}$ and an average scan time of $42 \mathrm{~min}$ per sample.

\section{$\mu C T$ evaluation of the epiphyseal cartilage}

Using the post-contrast scans, contour lines were drawn around a range of interest (ROI) that included the cartilage overlying the medial tibial plateau as described earlier [24,28]. Other ROIs were drawn and analyzed on this central midpoint of the articular surface in a manner corresponding to the standard histological evaluation techniques for the articular cartilage [29]. The length of the medial articular cartilage was measured and divided into 3 zones of equal length, and the cartilage volume was evaluated as described elsewhere [24].

\section{Cartilage histology}

After the EPIC $\mu \mathrm{CT}$ imaging of the articular cartilage was completed, six tibias were randomly chosen and placed in $10 \%$ neutral buffered formalin for $72 \mathrm{~h}$ prior to demineralization for 8 days in Immunocal (Decal Chemical Corp. Tillman, NY, USA). The tibias were then processed in paraffin and serially sectioned at $\sim 200-\mu \mathrm{m}$ intervals into 5 - $\mu \mathrm{m}$-thick sections for with hematoxylin and eosin, toluidine blue and safranin $\mathrm{O}$ to evaluate cartilage damage using the method suggested in the literature $[24,29,30]$. Here, we report the overall histology findings and zonal analysis of the cartilage thickness parameter, as evaluated using toluidine blue sections.

\section{$\mu C T$ evaluation of the cortical bone at the femoral mid-diaphysis}

$\mu \mathrm{CT}$ of the cortical bone mid-diaphysis was conducted on both the left and right femurs using a $\mu \mathrm{CT}-100^{\circ}$ computed tomography system and previously described method [31]. Sample scans were performed on 25 slices $(1$ slice $=10.5 \mu \mathrm{m})$ using high-resolution settings. The 
following parameters were evaluated bone mineral density, tissue volume, bone volume, bone marrow volume, cortical thickness, bone area, polar moment of inertia, maximal I value, minimal I value and $\mathrm{I}_{\mathrm{MAX}} / \mathrm{C}_{\mathrm{MAX}}$.

\section{Bone strength testing using the 3-point bending method}

Both the left and right femurs were mechanically tested with using an Instron materials testing machine (5543A, Instron Inc., Norwood, MA, USA). The femurs were positioned cranial side up across two lower contacts that had a span of 5-7 mm, with an upper contact centered between the lower contacts. The bone was broken in 3-point bending using a cross-head speed of $0.5 \mathrm{~mm} / \mathrm{min}$. During testing, force and displacement data were collected at a frequency of $200 \mathrm{~Hz}$ using the BlueHill 3 testing software, version 3.41 (Instron Inc). Force/displacement curves were generated, and ultimate force (maximum load), stiffness (maximum slope) and energy to fracture were recorded.

\section{Dynamic histomorphometry of the cortical bone}

The distal ends of the femoral cortical bone from the left and right femurs were collected after mechanical testing and embedded undecalcified in methylmethacrylate, cut into $20-\mu \mathrm{m}$-thick sections using a bone cutting system (Exakt Norderstedt, Germany) and evaluated for the new bone formation at the periosteal and endosteal envelope under UV light.

\section{Statistical analysis}

The data are reported as the means \pm standard deviations (SDs). Differences were tested for significance using 3-factor repeatedmeasures analysis of variance (ANOVA) with interactions (SigmaPlot, version 12.2, Systat Software, Chicago, IL, USA). Post hoc comparisons of means with a Bonferroni correction for multiple comparisons were performed only when interaction effects were significant. $P$ values less than 0.05 were considered statistically significant.

\section{Results}

\section{Animals}

All of the rats enrolled in the study showed a 15\% increase in body weight regardless of their treatment group. Neither the surgery nor treatment had an effect on animal health.

\section{Dynamic weight bearing}

The MM rats showed a different pattern of weight distribution compared with the sham controls. The weight-bearing load on the front feet of the MM rats was approximately $20 \%$ greater relative to sham controls at the 5-week time point, while both sham and MM rats showed similar weight bearing on the front feet at the end of study. The load-bearing capacity of the operated limbs of the MM rats did not increase despite the gain in body weight. The weight-bearing loads placed on the left hind leg were only moderately increased at 5 weeks in all 3 groups of MM rats regardless of treatment and stayed similar in all study groups throughout the 10-week study (Figure 1).

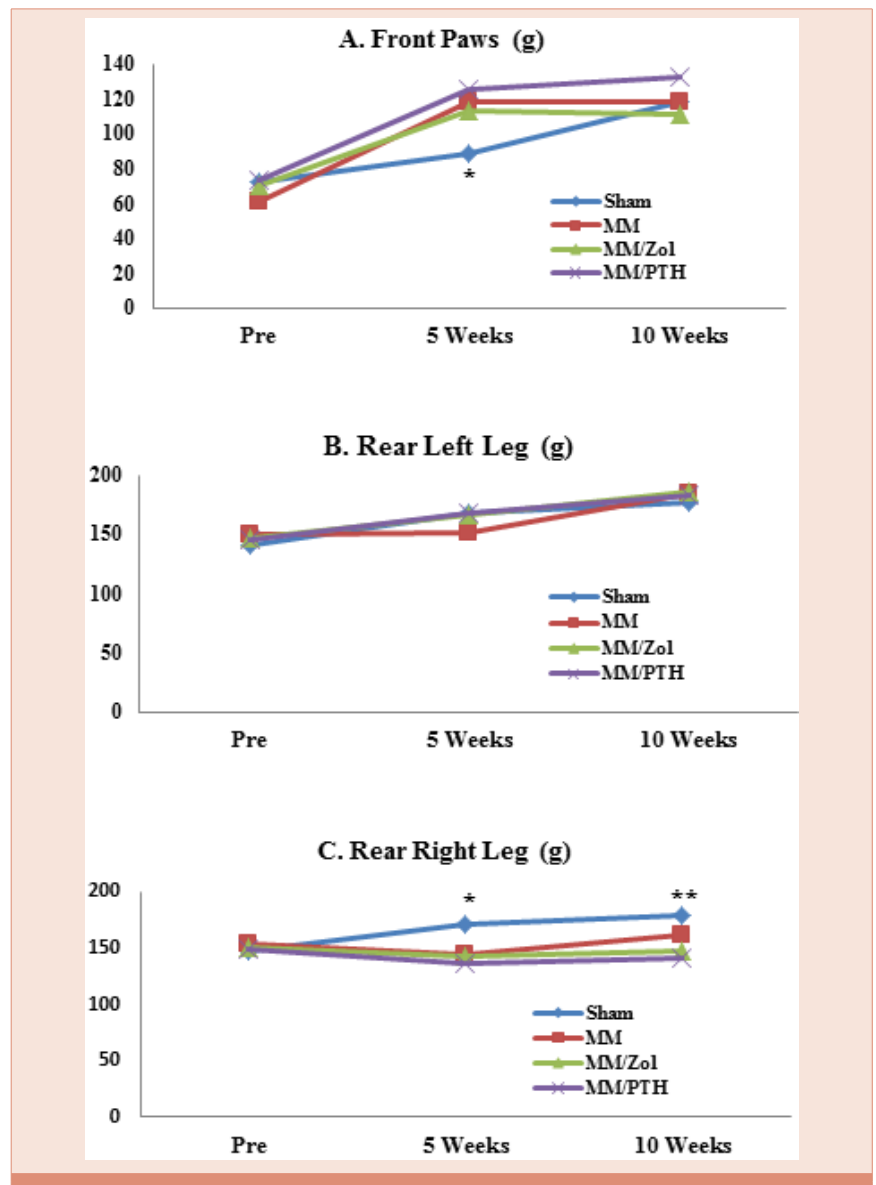

Figure 1: Figure 1 shows the change in the weight-bearing capacity of the front paws $(A)$, rear left leg $(B)$ and rear right leg $(C)$ in the Sham rats, MM rats, MM rats treated with zoledronate and MM rats treated with PTH. The weight-bearing load on the front paws in all three groups of MM rats was higher at the 5-week time point relative to the Sham controls. Additionally, the weight-bearing load on the rear right leg in all three groups of MM rats was lower at the 5- and 10-week time points relative to the Sham controls. ( $n=12$ rats/group; ${ }^{*} p<0.05$ vs MM, MM/Zol, MM/PTH and ${ }^{* *} p<0.05$ vs MM/ Zol and MM/PTH).

\section{EPIC $\mu C T$ evaluation of the articular cartilage at the medial tibial plateau}

The articular cartilage was significantly thicker in Zone 1, which borders the osteophytes, in all three groups of rats that received the MM surgery compared with the sham controls. The cartilage was either considerably thinner or completely missing in the lateral part of Zone 1 and in the entirety of Zone 2 in all 3 groups of MM rats compared with the sham controls Figure 2 A-D.

\section{Histology evaluation of the articular cartilage}

In the control sham rats, the articular cartilage at the medial tibial plateau grew progressively thicker from the most medial zone 1 to the most lateral part zone 3 (Figure 3A). The mechanical imbalance induced by the MM surgery resulted in cartilage thickening at the most medial half of zone 1, next to the osteophytes, whereas the cartilage in zone 2 was thin and or completely missing in all MM rats (Figure 3B-D). 

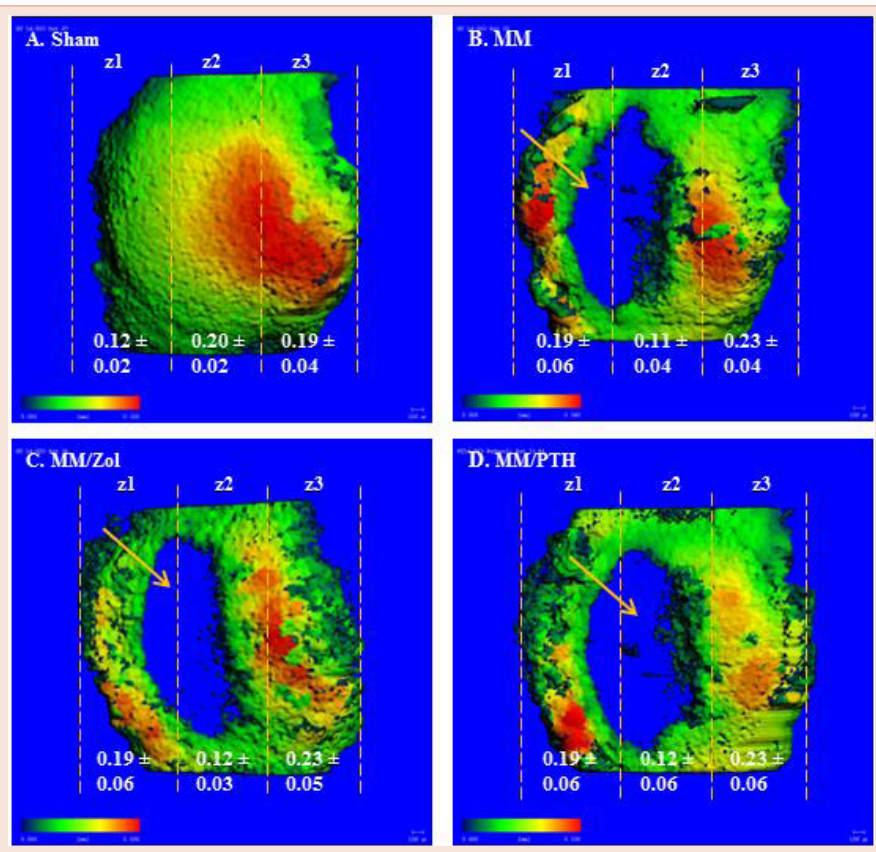

Figure 2 A-D: Figure 2 A-D shows the EPIC $\mu C T$ color thickness ("heat") images of the articular cartilage covering the medial tibial plateau. Large cartilage defects can be seen in Zones 1 and 2, as indicated by arrows. The most medial part of Zone 1 is thicker in all three MM groups compared with the Sham controls, while Zone 3 has a similar thickness in all four groups of rats. The numbers in parentheses indicate the mean \pm SD of cartilage volume in $\mathrm{mm}^{3}$ for each zone.

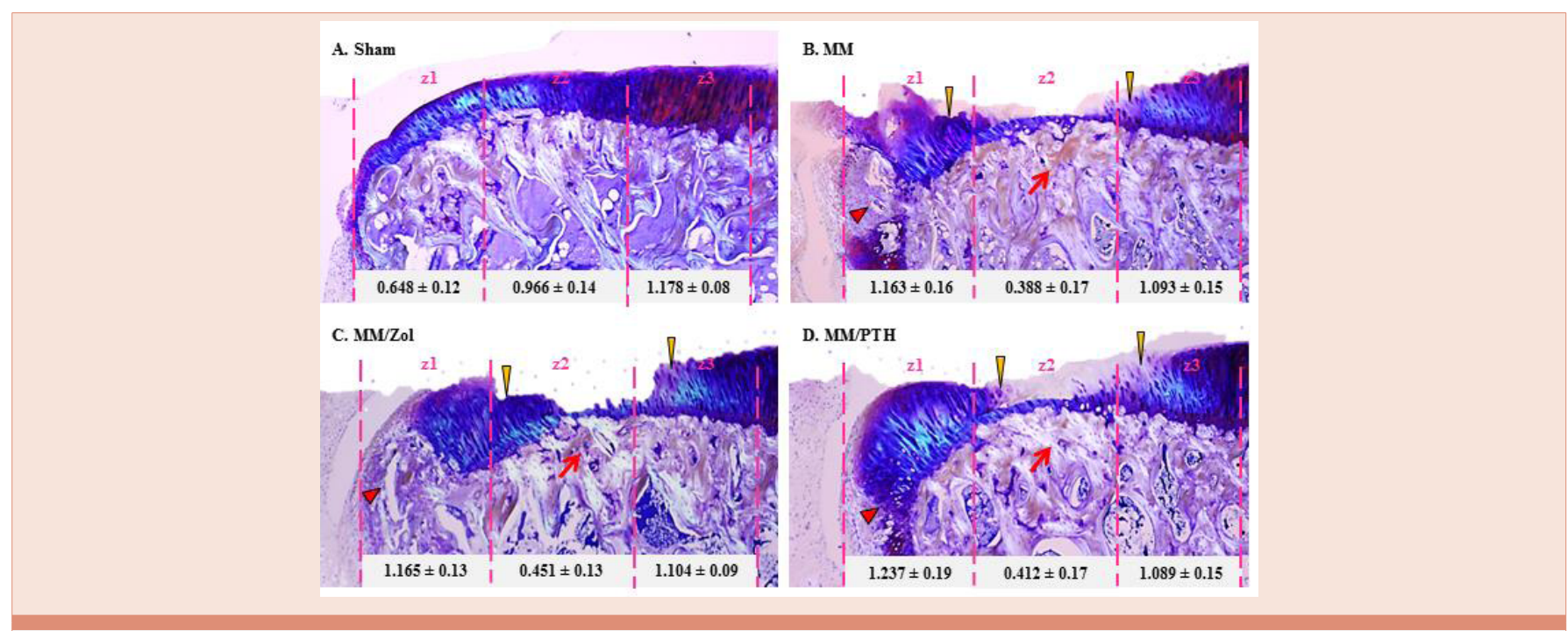

Figure 3 A-D: Figure 3A-D shows polarized images of the proximal tibial epiphysis stained with toluidine blue. All three groups of rats that received the MM surgery show significant cartilage deterioration, cartilage fibrillation (yellow arrowheads), and a thicker subchondral bone plate (red arrows) in Zones 1 and 2 . Osteophyte formation (red arrowheads) and thickening of the articular cartilage is evident in Zone 1 of them MM rats. The numbers in parentheses indicate the mean \pm SD of cartilage thickness in $\mu \mathrm{m}$ for each zone.

\section{Cortical bone geometry at the femoral mid-diaphysis}

The $\mu \mathrm{CT}$ results confirmed that the left and right femurs of the sham rats had similar cortical bone properties at the femoral middiaphysis. The left femurs of the MM rats showed cortical bone geometry similar to that of the left femurs of the sham rats. However, the right femurs of the MM rats showed lower values for cortical bone properties compared with the right femurs of the sham rats but also exhibited somewhat lower values for all measured parameters compared with the contralateral left femurs. Both femurs from zoledronate and PTH treated rats showed slightly higher values for cortical bone parameters relative to the MM controls. The left femurs of the rats treated with zoledronate and PTH showed somewhat higher values for cortical bone properties compared to corresponding right femurs (Table 1). 
Table 1: Table 1 shows the $\mu \mathrm{CT}$ analysis of the cortical bone at the femoral mid-diaphysis (midshaft) for both the left $(L)$ and right $(R)$ femurs. The following parameters were evaluated: bone mineral density $\left(\mathrm{BMD} ; \mathrm{mg} / \mathrm{cm}^{3}\right)$, tissue volume (T. Volume; $\left.\mathrm{mm}^{3}\right)$, bone volume $\left(B\right.$. Volume; $\left.\mathrm{mm}^{3}\right)$, bone $\mathrm{marrow}$ volume $\left(\mathrm{B} . \mathrm{M}\right.$. Volume; $\left.\mathrm{mm}^{3}\right)$, cortical thickness $(C$. Thickness; $\mathrm{mm})$, bone area $\left(B\right.$. Area; $\left.\mathrm{mm}^{2}\right)$, polar moment of inertia $\left(\mathrm{pMol} ; \mathrm{mm}^{4}\right), \mathrm{maximal}^{\mathrm{I}}$ value $\left(\mathrm{I}_{\mathrm{MAx}} ; \mathrm{mm}^{4}\right), \mathrm{minimal}^{\mathrm{I}}$ value $\left(\mathrm{I}_{\mathrm{MIN}} ; \mathrm{mm}^{4}\right)$, and $\mathrm{I}_{\mathrm{MAX}} / \mathrm{C}_{\mathrm{MAX}}\left(\mathrm{mm}^{3}\right)$.

\begin{tabular}{|c|c|c|c|c|c|c|}
\hline \multirow[b]{2}{*}{ Parameter } & \multirow[b]{2}{*}{ Unit } & \multirow[b]{2}{*}{ Leg } & \multicolumn{2}{|c|}{ STUDY } & \multicolumn{2}{|c|}{ GROUPS } \\
\hline & & & Sham & MM & MM/Zol & MM/PTH \\
\hline \multirow[t]{2}{*}{ BMD } & $\mathrm{mg} / \mathrm{mm}^{3}$ & $L$ & $1367.54 \pm 23.23$ & $1371.21 \pm 20.51$ & $1389.00 \pm 22.20$ & $1356.92 \pm 23.25$ \\
\hline & & $R$ & $1342.94 \pm 16.81$ & $1332.36 \pm 9.82$ & $1338.04 \pm 11.87$ & $1329.47 \pm 13.20$ \\
\hline \multirow[t]{2}{*}{ T. Volume } & $\mathrm{mm}^{3}$ & $L$ & $6.38 \pm 0.44$ & $6.08 \pm 0.37$ & $6.27 \pm 0.32$ & $6.40 \pm 0.29$ \\
\hline & & $R$ & $6.37 \pm 0.40$ & $6.04 \pm 0.55$ & $6.05 \pm 0.43$ & $6.18 \pm 0.32$ \\
\hline \multirow[t]{2}{*}{ B. Volume } & $\mathrm{mm}^{3}$ & $L$ & $3.05 \pm 0.15$ & $2.93 \pm 0.10$ & $3.06 \pm 0.17$ & $3.15 \pm 0.14$ \\
\hline & & $R$ & $2.99 \pm 0.19$ & $2.89 \pm 0.23$ & $2.93 \pm 0.17$ & $3.04 \pm 0.13$ \\
\hline \multirow[t]{2}{*}{ BM. Volume } & $\mathrm{mm}^{3}$ & $L$ & $3.13 \pm 0.31$ & $3.15 \pm 0.28$ & $3.21 \pm 0.19$ & $3.25 \pm 0.17$ \\
\hline & & $R$ & $2.98 \pm 0.23$ & $3.12 \pm 0.34$ & $3.13 \pm 0.28$ & $3.14 \pm 0.21$ \\
\hline \multirow[t]{2}{*}{ Co. Th } & $\mathrm{mm}$ & $L$ & $0.79 \pm 0.05$ & $0.77 \pm 0.01$ & $0.81 \pm 0.03^{a}$ & $0.82 \pm 0.02^{\mathrm{a}}$ \\
\hline & & $R$ & $0.79 \pm 0.03$ & $0.75 \pm 0.01$ & $0.78 \pm 0.02^{\mathrm{a}}$ & $0.80 \pm 0.01^{a}$ \\
\hline \multirow[t]{2}{*}{ B. Area } & $\mathrm{mm}^{2}$ & $L$ & $7.24 \pm 0.37$ & $6.96 \pm 0.24$ & $7.25 \pm 0.21^{a}$ & $7.47 \pm 0.34^{\mathrm{a}}$ \\
\hline & & $R$ & $7.19 \pm 0.26$ & $6.71 \pm 0.22$ & $6.95 \pm 0.21^{\mathrm{a}}$ & $7.21 \pm 0.30^{\mathrm{a}}$ \\
\hline \multirow[t]{2}{*}{ PMol } & $\mathrm{mm}^{4}$ & $L$ & $16.36 \pm 1.60$ & $15.46 \pm 1.41^{\#}$ & $16.21 \pm 1.70^{\#}$ & $17.15 \pm 1.69^{\#}$ \\
\hline & & $R$ & $16.28 \pm 1.13$ & $14.40 \pm 1.14^{*}$ & $14.95 \pm 1.72$ & $16.01 \pm 1.58$ \\
\hline \multirow[t]{2}{*}{$\mathrm{I}_{\operatorname{MAX}}$} & $\mathrm{mm}^{4}$ & $L$ & $10.18 \pm 1.11$ & $9.71 \pm 0.98^{\#}$ & $10.21 \pm 0.94^{\#}$ & $10.58 \pm 1.21^{\#}$ \\
\hline & & $R$ & $10.18 \pm 0.68$ & $8.79 \pm 0.70^{*}$ & $9.26 \pm 1.08$ & $9.68 \pm 1.02$ \\
\hline \multirow[t]{2}{*}{$\mathrm{I}_{\mathrm{MIN}}$} & $\mathrm{mm}^{4}$ & $L$ & $6.18 \pm 0.54$ & $5.75 \pm 0.49$ & $5.99 \pm 0.85$ & $6.57 \pm 0.54^{a}$ \\
\hline & & $R$ & $6.17 \pm 0.53$ & $5.61 \pm 0.53$ & $5.70 \pm 0.72$ & $6.34 \pm 0.65^{\mathrm{a}}$ \\
\hline \multirow[t]{2}{*}{$\mathrm{I}_{\text {MAX }} / \mathrm{C}_{\text {MAX }}$} & $\mathrm{mm}^{3}$ & $L$ & $4.53 \pm 0.35$ & $4.36 \pm 0.28$ & $4.58 \pm 0.34$ & $4.67 \pm 0.35$ \\
\hline & & $R$ & $4.45 \pm 0.22$ & $4.10 \pm 0.24$ & $4.30 \pm 0.40$ & $4.38 \pm 0.30$ \\
\hline
\end{tabular}

\section{Cortical bone strength}

The left femurs of all 4 study groups showed similar maximum load parameters. The right femurs of the rats that underwent MM surgery displayed significantly lower maximum loads and energy to break values compared with the contralateral left femurs of the same study group and the right femurs of the sham rats (Figure 4).

\section{Dynamic histomorphometry of the cortical bone}

The rats in the sham group showed a similar intensity of bone mineralization in both the left and right femurs and at both cortical envelopes. The rats that underwent MM surgery showed less intensive mineralization of the right (operated) legs compared with the contralateral left legs of the same dosing group. Additionally, the rats treated with PTH showed the most intense mineralization at both cortical envelopes while the zoledronate-treated rats showed minimal bone formation at the endosteal envelope in both femurs (Figure 5).

\section{Discussion}

Animal disease models for testing novel osteoarthritis therapies are the gold standard in the preclinical phase of drug development. Despite improvements in animal experimentation, technological advances and biomarkers, too many unnecessary and poorly designed animal studies are still being conducted, yielding redundant and misleading results that have not been clinically confirmed. Although the overall benefit of such studies to the medical community is negligible, the lack of standardized animal models, study designs and defined endpoints continues to enable the publishing of pointless data.

The recent study conducted by our group was one of a very few publications to show the lack of efficacy of antiresorptive and anabolic therapy on the key indicators of OA in a preclinical model of PTOA [24]. As summarized in this manuscript, study results reveal that neither zoledronate nor PTH had a substantial effect on cartilage deterioration, osteophyte formation, osteosclerosis and joint functionality. Similar to our findings, the clinical data fail to demonstrate a clear benefit of anabolic and antiresorptive therapies in OA patients [21,22] despite decades of the use of these agents to treat patients with skeletal maladies $[32,33]$. The animal models of bone diseases and the biomarkers of bone metabolism, mass and structure are highly predictive of clinical outcomes $[34,35]$. Similarly, animal models of OA also seem to closely replicate the pathophysiology of human disease and are endorsed by the OARSI governing body [23]. Therefore, the discrepancy between the preclinical results and the clinical findings regarding benefits of bisphosphonates and PTH 


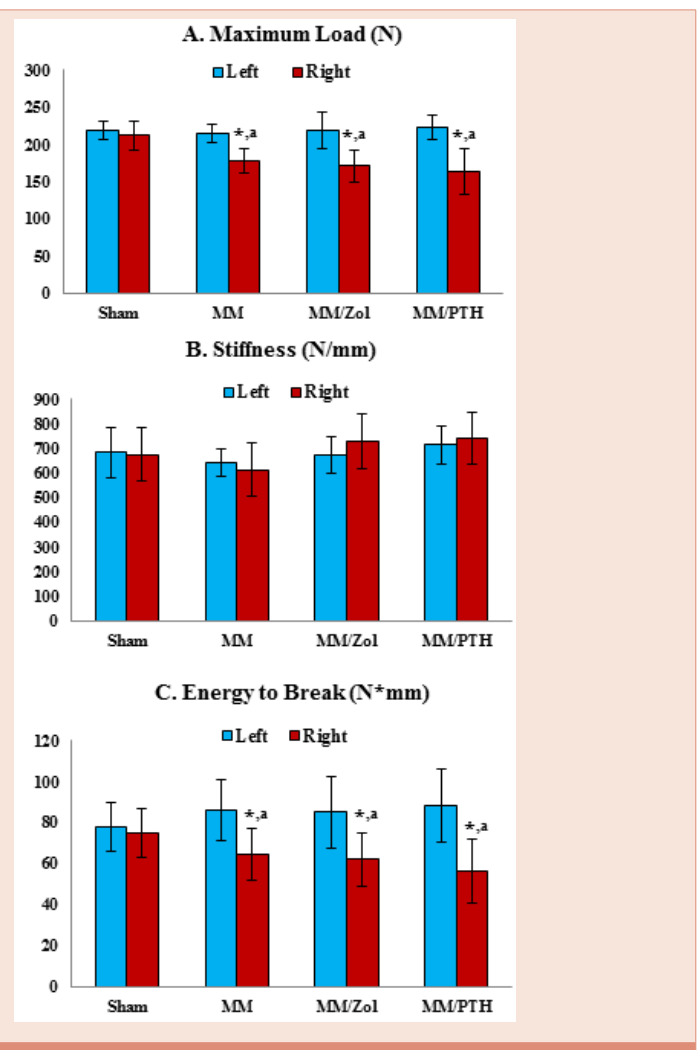

Figure 4: Figure 4 shows the change in maximum load (A), stiffness (B) and energy to break $(C)$ in the Sham rats, MM rats, MM rats treated with zoledronate and MM rats treated with PTH. The maximum load and energy to break parameters of the right femurs were decreased in all of the rats that underwent $\mathrm{MM}$ surgery relative to the right femurs of the sham controls. There was also a significant difference in the maximum load and energy to break parameters between the left and the right femurs of all rats that underwent MM surgery. ( $n=12$ rats/group; ${ }^{*} p<0.05$ vs the right femurs of the Sham group; ${ }^{a} p<0.05$ vs the left femurs of the same treatment group).

to treat $\mathrm{OA}$ require further evaluation. Based on the literature, we hypothesized that both therapies would maintain or improve the strength of the cortical bone in the OA limb and that the bones would thereby remain fit to withstand mechanical loads and provide a foundation implementing physical therapy. The published literature unequivocally indicates that therapy with PTH and bisphosphonates increases cortical bone mass and strength during physiologic loading and that the addition of physical exercise further promotes the drugs' effects on the cortical bone [36,37]. Contrariwise, in animal models with decreased load-bearing activity, anabolic and antiresorptive therapy seems to be less effective $[38,39]$, proving the "mechanostat" theory that bone mass and architecture are regulated in response to the local strains generated in the local tissue by functional loading $[40,41]$. The results of this study show that partial disuse and reduced weight-bearing loads negatively impact the cortical bone geometry of the operated leg, as all 3 groups of osteoarthritic rats consistently exhibited somewhat lower values for cortical bone denominators compared with the contralateral left leg. Even though decline in cortical bone qualities of the operated leg was rather small and reached the significant difference only for $\mathrm{pMoI}$ and $\mathrm{I}_{\mathrm{MAX}}$ parameters, the change seems to be sufficient to significantly affect cortical bone strength. Also, sedentary life style of rats in laboratory condition and different biomechanics in quadrupeds can minimize the change in cortical bone remodeling imposed by diminished capacity of osteoarthritic leg to withstand the mechanical loads and that should be taken into account when extrapolating these data to human subjects.

Similar to our findings, previous studies have shown that injured, osteoarthritic legs have a diminished capacity to withstand mechanical loads $[42,43]$. Here, we demonstrate that even moderate disuse over long periods has a deteriorating effect on cortical bone energy to break and maximal loads and that neither anabolic nor antiresorptive treatment can fully compensate for lack of physiological weightbearing loads. Although cortical bone geometry and stiffness (the ability of bone to resist deformation under a given load) is preserved with PTH and, to a lesser extent, with zoledronate, those therapies fail to significantly improve bone strength, perhaps because of impaired mineralization and changes in the material properties of the cortical bone [44].

The mechanical loading that occurs with weight-bearing exercise is a potent anabolic stimulus of muscle, bone, and cartilage and is
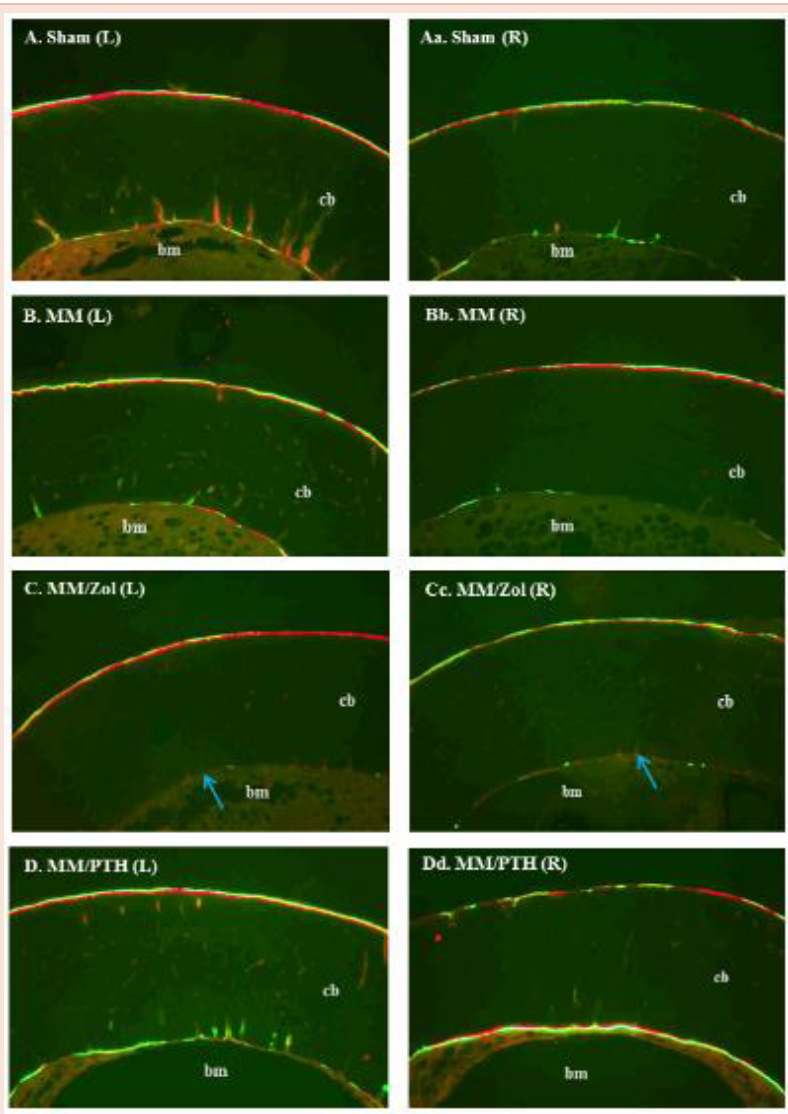

Figure 5: Figure 5 shows UV images of cortical bone from left $(\mathrm{L})$ and right (R) femurs. The right femurs of the rats that underwent MM surgery show less intense mineralization at both cortical envelopes. The blue arrows indicate minimal bone formation at the endosteal envelope in the zoledronate-treated rats. The rats treated with PTH show the most intense formation of both the periosteal and endosteal envelope. (bm - bone marrow; $\mathrm{cb}$ - cortical bone). 
essential for the health of the musculoskeletal system [9-11,45]. The data from this study led us to conclude that patients with acute traumatic cartilage injury will benefit most from the early implementation of physical therapy and moderate exercise [45-48], which should provide the optimal milieu for simultaneous treatment with other therapies (anti-inflammatory, regenerative, nutrient and vitamin, and bone therapies) to support healing of the articular cartilage and subchondral bone end to ensure that the mechanical functions of the injured joint are restored.

In recent years, the regulatory and scientific community has imposed stringent rules to ensure that the well-being of laboratory animals and that the 3 Rs paradigm is implemented [25,49]. It has been estimated that 25 million animals are used in research every year [50]. To further improve the usefulness of animal studies investigators should carefully select a suitable disease model and design the study of appropriate duration taking into account the slow metabolism of the cartilage and subchondral bone to allow the complete assessment of joint functionality. Additionally, several independent methods should be mandatory for every in vivo study to evaluate key attributes of cartilage and bone physiology, including imaging and histological evaluation of cartilage and bone, histochemistry and serum biomarkers. The lack of appropriate skills, technologies or funding should not be an excuse to compromise and run incomplete and poorly designed studies. Publishing "negative" data, even in the form of summary, should be encouraged to further promote information sharing and deter scientists from conducting unnecessary and redundant work. To that end, scientific journals and conferences should enforce stricter rules and guidelines for publishing data from in vivo studies to ensure scientific excellence and the proper use of laboratory animals.

\section{Conclusions}

The various methods utilized in this study showed that aggressive treatment with zoledronate and PTH was not sufficient to prevent or correct the deterioration of the hyaline cartilage, osteophyte formation and the mechanical incapacity of the osteoarthritic knee. Additionally, partial disuse of the osteoarthritic leg over long periods weakens cortical bone strength, and neither anabolic nor antiresorptive treatment could compensate for the lack of physiological weight-bearing loads. Our data suggest that the quick restoration of the mechanical function of the injured knee is a mandatory first step to prevent cartilage deterioration and support the recovery of joint structures and that a multi-therapeutic approach is warranted to treat various aspects of post-traumatic OA.

\section{Author contributions}

All authors made substantial contributions to study design, analysis, and interpretation of data, drafting of the manuscript, and editing, making important intellectual contributions to the content of this article.

\section{Role of Funding Source}

This study was supported by Pfizer Consumer Healthcare. The study's sponsor had no role in the study design, data collection, and data analysis and data interpretation and did not participate in the manuscript's writing or submission.

\section{Competing Interest}

All Pfizer authors are regular employees and have no conflicts of interest to declare. Isabela Bagi was a summer intern at Pfizer and has no conflict of interest to declare.

\section{Acknowledgement}

The authors thank Michael Wadanoli, Adam Murphy and Dean Wilkie for their excellent technical assistance.

\section{References}

1. Bijlsma JW, Berenbaum F, LaFeber FP (2011) Osteoarthritis: an update with relevance for clinical practice. Lancet 377: 2115-2126.

2. Felson DT, Lawrence RC, Dieppe PA, Hirsch R, Helmick CG, et al. (2000) Osteoarthritis: new insights. part 1: The disease and its risk factors. Ann Intern Med 133: 635-646.

3. Britton R (2009) The economic burden of osteoarthritis. Am J Manag Care 15: 230-235.

4. Brown TD, Johnston RC, Saltzman CL, Marsh JL, Buckwalter JA (2006) Posttraumatic osteoarthritis: a first estimate of incidence, prevalence, and burden of disease. J Orthop Trauma 20: 739-744.

5. Carter DR, Wong M, Orr TE (1991) Musculoskeletal ontogeny, phylogeny, and functional adaptation. J Biomech 24: 3-16.

6. Helminen HJ, Saamanen A, Jurvelin J, Kiviranta I, Parkkinen JJ, et al. (1992) The effect of loading on articular cartilage. Duodecim 108: 1097-1107.

7. Lotz M (2001) Cytokines in cartilage injury and repair [review]. Clin Orthop 391: S108-115

8. Hunziker EB (2002) Articular cartilage repair: basic science and clinical progress. A review of the current status and prospects. Osteoarthritis Cartilage 10: 432-463.

9. Griffin TM, Guilak F (2005) The role of mechanical loading in the onset and progression of osteoarthritis. Exerc Sport Sci Rev 33: 195-200.

10. Robling AG, Turner $\mathrm{CH}$ (2009) Mechanical signaling for bone modeling and remodeling. Crit Rev Eukaryot Expr 19: 319-338.

11. Yokota H, Leong DJ, Sun HB (2011) Mechanical loading: bone remodeling and cartilage maintenance. Curr Osteoporos Rep 9: 237-242

12. Lange AK, Vanwanseele B, Fiatarone Singh MA (2008) Strength training for treatment of osteoarthritis of the knee: a systematic review. Arthritis Rheum 59: $1488-1494$

13. Bennell KL, Hinman RS (2011) A review of the clinical evidence for exercise in osteoarthritis of the hip and knee. J Sci Med Sport 14: 4-9.

14. Hochberg MC, Altman RD, April KT, Benkhalti M, Guyatt G, et al. (2012) American College of Rheumathology 2012 recommendations for the use of nonpharmacologica and pharmacologic therapies in osteoarthritis of the hand, hip, and knee. Arthritis Care Res 64: 465-474.

15. Felson DT, Neogi T (2004) Osteoarthritis: is it a disease of cartilage or of bone? Arthritis Rheum 50: 341-344.

16. Bailey AJ, Mansell JP, Sims TJ, Banse X (2004) Biochemical and mechanical properties of subchondral bone in osteoarthritis. Biorheol 41: 349-358.

17. Muehleman C, Green J, Williams JM, Kuettner KE, Thonar EJ, et al. (2002) The effect of bone remodeling inhibition by zoledronic acid in an animal model of cartilage matrix damage. Osteoarthritis Cartilage 10: 226-233.

18. Siebelt M, Waarsing JH, Groen HC, Müller C, Koelewijn SJ, et al. (2014) Inhibited osteoclastic bone resorption through alendronate treatment in rats reduces severe osteoarthritis progression. Bone 66: 163-170.

19. Chang JK, Chang LH, Hung SH, Wu SC, Lee HY, et al. (2009) Parathyroid 
hormone 1-34 inhibits terminal differentiation of human articular chondrocytes and osteoarthritis progression in rats. Arthritis Rheum 60: 3049-3060.

20. Sampson ER, Hilton MJ, Tian Y, Chen D, Schwarz EM, et al. (2011) Teriparatide as a chondroregenerative therapy for injury-induced osteoarthritis. Sci Transl Med 3: 101ra93.

21. Bingham III CO, Buckland-Wright JC, Garnero P, Cohen SB, Dougados $\mathrm{M}$, et al. (2006) Risedronate decreases biochemical markers of cartilage degradation but does not decrease symptoms or slow radiographic progression in patients with medial compartment osteoarthritis of the knee: results of the two-year multinational knee osteoarthritis structural arthritis study. Arthritis Rheum 54: 3494-3507.

22. Neogi T, Nevitt MC, Ensrud KE, Bauer D, Felson DT (2008) The effect of alendronate on progression of spinal osteophytes and disc-space narrowing. Ann Rheum Dis 67: 1427-1430.

23. Bendele A (2001) Animal models of osteoarthritis. J Musculoskelet Neuronal Interact 1: 363-376.

24. Bagi CM, Berryman E, Zakur DE, Wilkie D, Andresen CJ (2015) Effect of Antiresorptive and anabolic bone therapy on development of osteoarthritis in a posttraumatic rat model of OA. Arthritis Res Ther 17: 315

25. National Research Council (2011) The Guide for the Care and Use of Laboratory Animals. Washington, National Academy Press.

26. Bagi CM, Zakur DE, Berryman E, Andresen CA, Wilkie D (2015) Correlation between $\mu \mathrm{CT}$ imaging, histology and functional capacity of the osteoarthritis knee in the rat model of osteoarthritis. J Transl Med 13: 276.

27. Xie L, Lin AS, Guldberg RE, Levenston ME (2010) Nondestructive assessment of SGAG content and distribution in normal and degraded rat articular cartilage via EPIC-microCT. Osteoarthritis Cartilage 18: 65-72.

28. Kokkonen HT, Jurvelin JS, Tiitu V, Töyräs J (2011) Detection of mechanica injury of articular cartilage using contrast enhanced computed tomography. Osteoarthritis Cartilage 19: 295-301

29. Gerwin N, Bendele AM, Glasson S, Carlson CS (2010) The OARSI histopathology initiative - recommendations for histological assessments of osteoarthritis in the rat. Osteoarthritis Cartilage 18: S24-34

30. Pastoureau PC, Hunziker EB, Pelletier JP (2010) Cartilage, bone and synovial histomorphometry in animal models of osteoarthritis. Osteoarthritis Cartilage 18: S106-112

31. Bagi CM, Hanson N, Andresen C, Pero R, Lariviere R, et al. (2006) The use of micro-CT to evaluate cortical bone geometry and strength in nude rats: correlation with mechanical testing, pQCT and DXA. Bone 38: 136-144.

32. Black DM, Delmas PD, Eastell R, Reid IR, Boonen S, et al. (2007) Once yearly zoledronic acid for treatment of postmenopausal osteoporosis. N Engl J Med 356: 1809-1822.

33. Han SL, Wan SL (2012) Effect of teriparatide on bone mineral density and fracture in postmenopausal osteoporosis: meta-analysis of randomised controlled trials. Int J Clin Pract 66: 199-209.

34. Gasser JA, Ingold P, Venturiere A, Shen V, Green JR (2008) Long-term protective effects of zoledronic acid on cancellous and cortical bone in the ovariectomized rat. J Bone Miner Res 23: 544-551.

35. Kneissel M, Boyde A, Gasser JA (2001) Bone tissue and its mineralization in aged estrogen-depleted rats after long-term intermittent treatment with parathyroid hormone (PTH) analog SDZ PTS 893 or human PTH (1-34). Bone 28: 237-250.

36. Fuchs RK, Shea M, Durski SL, Winters-Stone KM, Widrick J, et al. (2007) Individual and combined effects of exercise and alendronate on bone mass and strength in ovariectomized rats. Bone 41: 290-296.

37. Sugiyama T, Saxon LK, Zaman G, Moustafa A, Sunters A, et al. (2008) Mechanical loading enhances the anabolic effects of intermittent parathyroid hormone (1-34) on trabecular and cortical bone in mice. Bone 43: 238-248.

38. Turner RT, Lotinun S, Hefferan TE, Morey-Holton E (20061) Disuse in adult male rats attenuates the bone anabolic response to a therapeutic dose of parathyroid hormone. J Appl Physiol 101: 881-886.

39. Macias BR, Swift JM, Nilsson MI, Hogan HA, Bouse SD, et al. (2012) Simulated resistance training, but not alendronate, increases cortical bone formation and suppresses sclerostin during disuse. J Appl Physiol 112: 918925.

40. Frost HM (1996) Perspectives: a proposed general model of the "mechanostat" (suggestions from a new skeletal-biologic paradigm). Anat Rec 244: 139-147.

41. Sugiyama T, Price JS, Lanyon LE (2010) Functional adaptation to mechanical loading in both cortical and cancellous bone is controlled locally and is confined to the loaded bones. Bone 46: 314-321.

42. Fernihough J, Gentry C, Malcangio M, Fox A, Rediske J, et al. (2004) Pain related behaviour in two models of osteoarthritis in the rat knee. Pain 112: 83-93.

43. Allen KD, Mata BA, Gabr MA, Huebner JL, Adams SB, et al. (2012) Kinematic and dynamic gait compensations resulting from knee instability in a rat model of osteoarthritis. Arthritis Res Ther 14: R78.

44. Cheng Z, Yao W, Zimmermann EA, Busse C, Ritchie RO, et al. (2009) Prolonged treatments with antiresorptive agents and PTH have different effects on bone strength and the degree of mineralization in old estrogendeficient osteoporotic rats. J Bone Miner Res 24: 209-220.

45. Bader DL, Salter DM, Chowdhury TT (2011) Biomechanical influence of cartilage homeostasis in health and disease. Arthritis 2011: 979032.

46. Englund $M(2010)$ The role of biomechanics in the initiation and progression of OA of the knee. Best Pract Res Clin Rheumatol 24: 39-46.

47. Iwamoto J, Sato Y, Takeda T, Matsumoto H (2011) Effectivness of exercise for osteoarthritis of the knee: a review of the literature. World J Orthop 18: 37-42.

48. Teichtahl AJ, Wluka AE, Wijethilake P, Wang Y, Ghasem-Zadeh A, et al. (2015) Wolff's law in action: a mechanism for early knee osteoarthritis. Arthritis Res Ther 17: 207.

49. (2013) Animal research: a balancing act. Nat Med 19: 1191.

50. Cates CC, Couto MA (2014) In vivo physiological research in the US: regulatory and ethical issues. Open Access Anim Physiol 2015: 1-11.

Copyright: @ 2016 Bagi CM, et al. This is an open-access article distributed under the terms of the Creative Commons Attribution License, which permits unrestricted use, distribution, and reproduction in any medium, provided the original author and source are credited. 